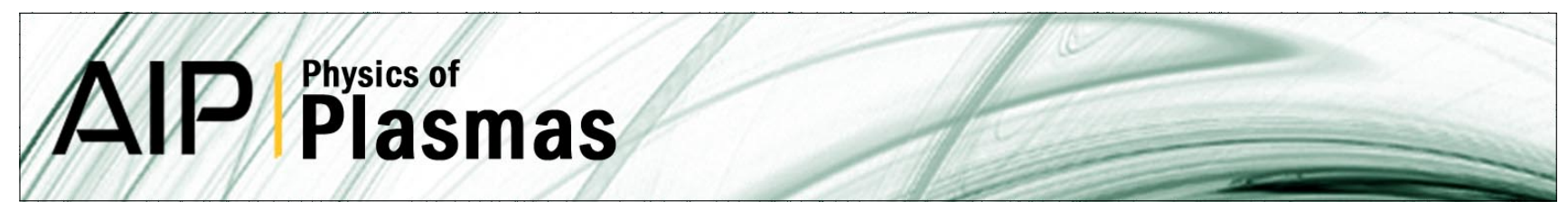

\title{
Periodically oscillating plasma sphere
}

J. Park, R. A. Nebel, S. Stange, and S. Krupakar Murali

Citation: Phys. Plasmas 12, 056315 (2005); doi: 10.1063/1.1888822

View online: http://dx.doi.org/10.1063/1.1888822

View Table of Contents: http://pop.aip.org/resource/1/PHPAEN/v12/i5

Published by the American Institute of Physics.

\section{Additional information on Phys. Plasmas}

Journal Homepage: http://pop.aip.org/

Journal Information: http://pop.aip.org/about/about_the_journal

Top downloads: http://pop.aip.org/features/most_downloaded

Information for Authors: http://pop.aip.org/authors

\section{ADVERTISEMENT}

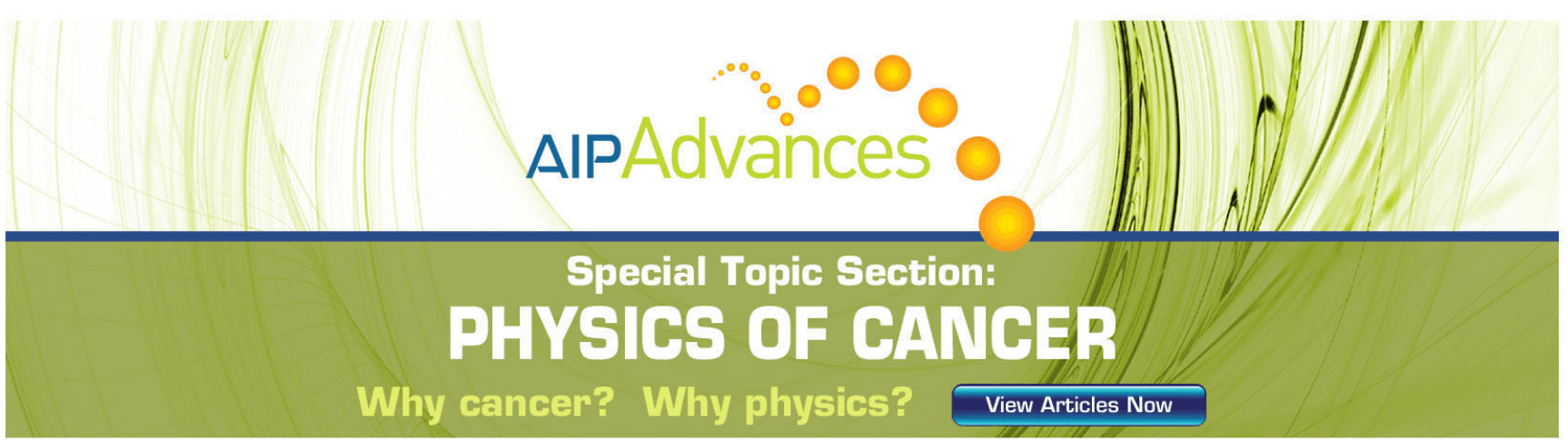




\title{
Periodically oscillating plasma sphere ${ }^{a)}$
}

\author{
J. Park, ${ }^{\text {b) }}$ R. A. Nebel, and S. Stange \\ Los Alamos National Laboratory, Los Alamos, New Mexico 87544 \\ S. Krupakar Murali \\ University of Wisconsin, Madison, Wisconsin 53706
}

(Received 2 December 2004; accepted 14 February 2005; published online 10 May 2005)

The periodically oscillating plasma sphere, or POPS, is a novel fusion concept first proposed by D. C. Barnes and R. A. Nebel [Fusion Technol. 38, 28 (1998)]. POPS utilizes the self-similar collapse of an oscillating ion cloud in a spherical harmonic oscillator potential well formed by electron injection. Once the ions have been phase-locked, their coherent motion simultaneously produces very high densities and temperatures during the collapse phase of the oscillation. A requirement for POPS is that the electron injection produces a stable harmonic oscillator potential. This has been demonstrated in a gridded inertial electrostatic confinement device and verified by particle simulation. Also, the POPS oscillation has been confirmed experimentally through observation that the ions in the potential well exhibit resonance behavior when driven at the POPS frequency. Excellent agreement between the observed POPS frequencies and the theoretical predictions has been observed for a wide range of potential well depths and three different ion species. Practical applications of POPS require large plasma compressions. These large compressions have been observed in particle simulations, although space charge neutralization remains a major issue. (C) 2005 American Institute of Physics. [DOI: 10.1063/1.1888822]

\section{INTRODUCTION}

Inertial electrostatic confinement (IEC) is a general term describing plasma confinement devices and was first proposed by Elmore et al., ${ }^{1}$ Farnsworth, ${ }^{2}$ and Hirsch $^{3}$ in the 1950s and 1960s. IEC devices use either electrostatic fields ${ }^{1-4}$ or a combination of electrostatic and magnetic fields ${ }^{5-8}$ to confine the ions. Typically, the confining fields are produced either by grids or by virtual cathodes, either in spherical or cylindrical geometry. The fields create an effective potential well that accelerates the ions and focuses them towards the center of the device, where fusion reactions can occur. Ions undergo multiple passes between the grid and the center and produce fusion reactions until they are absorbed by the grid or lose energy by collision processes. The ease of accelerating the ions to a fusion relevant energy range of $10-150 \mathrm{keV}$ in a table-top device was the basis for IEC's early success-it produced a steady-state neutron yield of 2 $\times 10^{10}$ neutrons/s using deuterium-tritium (D-T) fuels in the late 1960s. ${ }^{4}$

Though promising as practical neutron sources, existing IEC fusion devices operate at modest fusion yields, $\sim 0.01 \%$ or less of the input power. There are three principle causes for the modest yields. In a gridded system, where the confining fields are created by a physical grid, the main energy loss comes from particle loss to the grid. Typical grid transparencies are about $90 \%-95 \%$, resulting in ions being lost after 5-10 grid passes. Secondary electron emission from the grid further enhances the energy loss. IEC devices also suffer significant energy losses from ion-neutral charge-exchange

\footnotetext{
a) Paper NI2 5, Bull. Am. Phys. Soc. 49, 250 (2004).

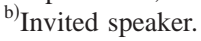

collisions since the neutrals are not confined by the potential well. Finally, ion-ion Coulomb collisions can cause a rapid relaxation of the desired beamlike ion distribution to a Maxwellian distribution. These collisions add angular momentum to the particles and cause the desired focus to expand. Consequently, either the energy cost of maintaining the beamlike ion distribution exceeds the fusion energy gain ${ }^{9,10}$ or the fusion power density becomes too small as the ion focus spreads. ${ }^{11}$

Solutions for these issues have been proposed with some degree of success. Gridless IEC devices such as a Penning trap $^{6}$ or a Polywell ${ }^{\mathrm{TM}}$ configuration ${ }^{5}$ can form an effective potential well by combining electrostatic and magnetic fields without a physical grid. Also, ion-beam assisted IEC devices $^{4}$ and virtual cathode systems ${ }^{12,13}$ have low operating background gas pressures that reduce the energy loss due to charge exchange and should improve fusion yields. However, the high energy cost of maintaining a beamlike ion energy distribution against Coulomb collisions remains, which makes it difficult to produce net fusion power.

A new electrostatic plasma equilibrium that can mitigate this problem has been proposed by Barnes and Nebel. ${ }^{14,15}$ This concept uses electron injection into a spherical device to produce a virtual cathode with a harmonic oscillator potential. In spherical geometry, the harmonic oscillator potential is produced by a radially constant electron density. An ion cloud immersed in the virtual cathode (referred to as the periodically oscillating plasma sphere, or POPS) will then undergo a harmonic oscillation with an oscillation frequency that is independent of the amplitude. By tuning the external radio-frequency (rf) electric fields to this naturally occurring mode, it is then possible to phase-lock the ion motion. Dur- 
ing the collapse phase of the coherent oscillation, all ions converge into the center with their maximum kinetic energies. The POPS concept relies on the coherent harmonic oscillation to simultaneously produce very high densities and temperatures rather than using beamlike ions.

An analytic solution for the POPS oscillation exists that has the remarkable property of maintaining the ions in local thermodynamic equilibrium at all times. ${ }^{14}$ This is because the phase space dynamics of the harmonic oscillator follow a circular path between the ion velocity and its radial displacement. The POPS oscillation can be described as a rigid rotor rotation in phase space with the velocity distribution and the radial density profile exchanging places every quarter period. ${ }^{16}$ If the radial density profile is Gaussian, the corresponding velocity distribution is Maxwellian and ion-ion collisions will not cause any further relaxation. This eliminates most of the energy loss due to Coulomb collisions and can greatly increase the fusion yield. In principle, it is possible to obtain the net energy gain required for fusion power generation. The spherical harmonic potential well needed for POPS can be created in either a gridded device or a Penning trap, since the ion equations for POPS are identical for both devices. Therefore, the practical embodiment of a POPS fusion device will most likely be based on the Penning trap due to its inherent low particle loss.

In this report, we describe experimental and theoretical investigations of POPS in a gridded IEC device. A detailed description of the experimental configuration is given in Sec. II. In Sec. III, experimental results are presented, including the formation of stable harmonic oscillator potential wells and the observation of POPS oscillations. Space charge neutralization issues are addressed in Sec. IV, which describes one-dimensional particle simulations. Discussion and conclusions are in Sec. V.

\section{EXPERIMENTAL SETUP}

The IEC device at Los Alamos National Laboratory, known as the intense neutron source-electrons (INS-e), consists of a 12 in. (o.d.) spherical vacuum vessel containing two concentric, highly transparent wire grids. A schematic diagram of the device is shown in Fig. 1. The vessel is double walled and water cooled, constructed of 304 stainless steel, and its vacuum pumping is provided by a 150 liter/s turbomolecular pump backed by a liquid nitrogen cold trap and a mechanical roughing pump. The base pressure typically reaches below $1 \times 10^{-8}$ torr without baking. The electron injection in the INS-e device comes from thermally emitting barium-impregnated tungsten cathodes. This eliminates the need for Paschen breakdown and reduces the operating gas pressure to between $(0.1-10) \times 10^{-6}$ torr, which is two to four orders of magnitude lower than other IEC devices. Additionally, the formation of the virtual cathode is independent of the fill gas. Several different fill gases are used in INS-e, including hydrogen, deuterium, helium, and neon. Although similar experimental conditions can be achieved in a Penning trap configuration, the gridded configuration was chosen for more flexibility in controlling the plasma parameters and better diagnostic access.

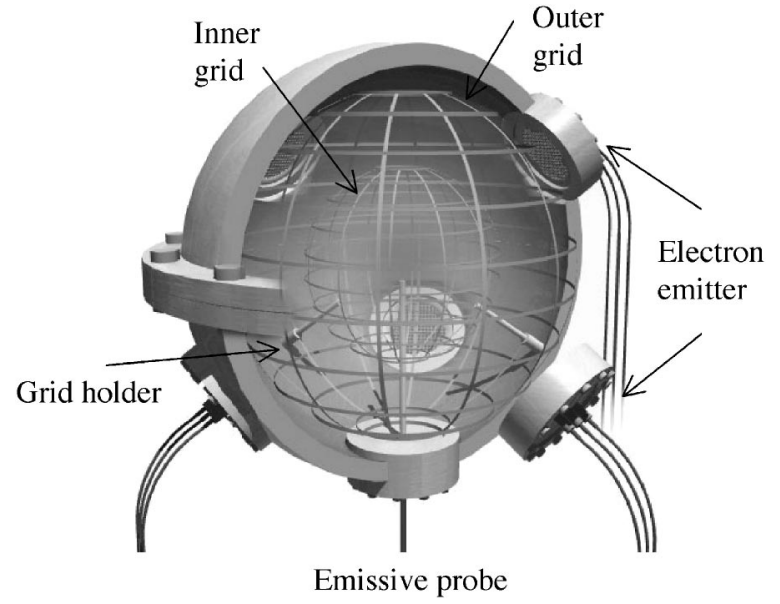

FIG. 1. Schematic of the INS-e device.

In INS-e, the grids are constructed of wires made of an alloy of 75\% tungsten and 25\% rhenium. A combination of three different wire sizes $(0.02,0.04$, and $0.08 \mathrm{~cm}$ diameter $)$ is used in order to achieve a high degree of transparency $(\sim 92 \%)$ and structural strength. The outer grid is $23 \mathrm{~cm}$ in diameter and the inner grid is $12.7 \mathrm{~cm}$ in diameter. The key parameter for the grid design is the grid spacing between the wires. The grid spacing of the inner grid is maintained below $1.0 \mathrm{~cm}$. Radial profile measurements of the plasma potential show that the plasma potential at the midpoint between the grid wires agrees to within $10 \%$ of the grid bias voltage. In comparison, the local effective Debye length is $\sim 1.8 \mathrm{~cm}$. A small grid spacing helps maintain the spherical symmetry of the plasma potential in the virtual cathode. The grid spacing for the outer grid is $\sim 2 \mathrm{~cm}$. There are three separate $\mathrm{dc}$ power systems for powering the electron emitters, the inner grid, and the outer grid independently. Additionally, two pulse generators (Directed Energy Inc., PVX-4140 and PVM-4150) with rise times of $\sim 25 \mathrm{~ns}$ are used in conjunction with the dc power systems to operate the INS-e in a pulsed mode.

The primary diagnostic for the INS-e is an emissive probe that measures the plasma potential and its temporal variation. The emissive probe utilizes a thoriated tungsten wire to enhance the thermal electron emission and is constructed following the method suggested by Siebenförcher and Schrittwieser. ${ }^{17}$ The probe can be moved along the axis to measure the radial profile of the plasma potential. The probe circuit allows a small leakage current, $\sim 10 \mu \mathrm{A}$, in order to obtain a temporal resolution of $\sim 0.1 \mathrm{~ms}$ without disturbing the surrounding plasmas. The accuracy of the plasma potential measurement has been verified using the inflection point method. ${ }^{18}$

\section{EXPERIMENTAL RESULTS}

POPS requires a stable virtual cathode where ions can undergo harmonic oscillations. In INS-e, the virtual cathode is created by electron injection from six emitters. As shown in Fig. 2, a stable virtual cathode has been created, but low gas pressures are required, typically in the range of 


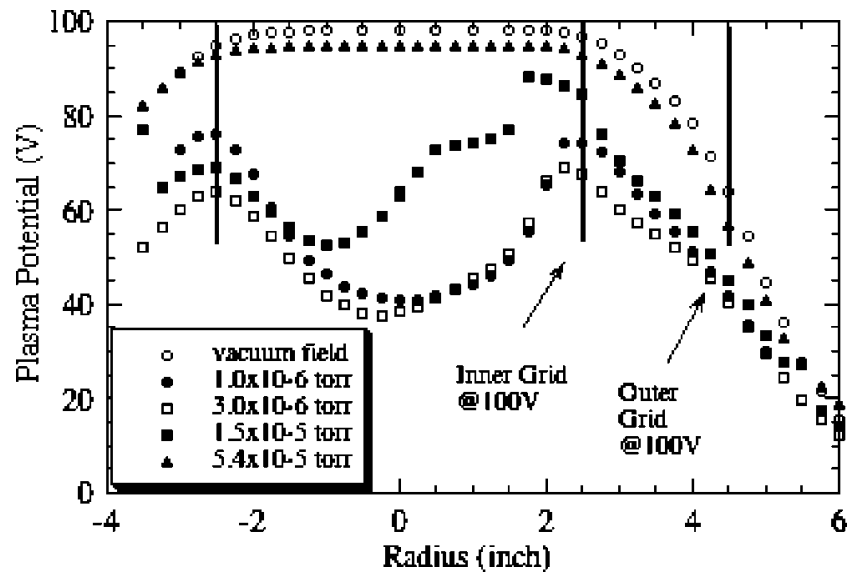

FIG. 2. Radial profiles of the plasma potential at various fill pressures. The bias voltages for the extractor grids, the outer grid, and the inner grid are all 100 V. (Reproduced from Ref. 12.)

$10^{-6}$ torr. $^{12}$ In steady-state operation, the ion density in the virtual cathode monotonically increases in time due to the ionization of the background gases and a practically infinite ion confinement time. ${ }^{13}$ This ion buildup results in the loss of the virtual cathode on a time scale comparable to the background ionization time, 0.5-5 ms. Therefore, pulsed power systems are used to bias the electron emitter and the inner grid in order to limit the ion buildup in the virtual cathode. Typical pulse durations are between 0.5 and $10 \mathrm{~ms}$, where the lower bound of the pulse duration is chosen to accommodate the slow temporal response of the emissive probe and the upper bound is chosen to match the decay of the virtual cathode. The pulse duration is sufficiently long to study the POPS oscillations, since it is many times longer than the POPS period, which is a few microseconds.

Two additional conditions are needed besides the stable virtual cathode in order to excite POPS oscillations. The first is to control the radial profile of the injected electrons, and thus the shape of the plasma potential. In particular, a flat radial electron density profile is required to produce a spherical harmonic oscillator potential well. At present, this is done by separately controlling the grid biases of the emitter grids, the inner grid, and the outer grid. The emitter bias controls the electron injection current, while the inner grid bias controls the electron energy. Together they vary the electron den- sity in the well and concurrently the potential well depth in the virtual cathode. On the other hand, the difference in the bias voltage between the inner grid and the outer grid creates either an accelerating or a retarding electric field that controls the angular momentum distribution of the injected electrons. Typically, a slight retarding potential and a modest injection current lead to the desired flat electron density profile. In practice, fine tuning of all three of the bias voltages is required to produce a flat electron density profile in INS-e.

The second condition to excite the POPS oscillation is to provide an external perturbation to phase-lock the ion motion. ${ }^{14,15,19}$ In this paper, an rf modulation of $5-10 \mathrm{~V}$ is applied to the inner grid during the high voltage pulse. With an rf perturbation applied to the inner grid, the ion dynamics in the virtual cathode are those of a driven harmonic oscillator, which is described by the Mathieu equations. ${ }^{20}$ At the fundamental resonance, where the external modulation frequency is twice the intrinsic harmonic oscillator frequency or POPS frequency, even infinitesimal perturbations drive the ion orbits unstable and eventually eject them from the virtual cathode. The ion ejection can limit the ion buildup caused ionization of the background gas and thus slow the decay of the virtual cathode. This resonance has been measured by monitoring the change in the virtual cathode decay time using an emissive probe, as shown in Fig. 3. ${ }^{19}$ Figure 3(a) shows the temporal evolution of the plasma potential as measured by the emissive probe at the center of the device. A significant delay in the loss of the virtual cathode was observed for a moderate rf modulation amplitude of $\sim 8 \mathrm{~V}$ at $350 \mathrm{kHz}$, compared to the case without rf modulation and with rf modulation at other frequencies. In Fig. 3(b), the delay time in the onset of the virtual cathode loss due to rf modulation is plotted as a function of the modulation frequency from $100 \mathrm{kHz}$ to $1 \mathrm{MHz}$, showing the fundamental resonance at $\sim 350 \mathrm{kHz}$ and the much broader half harmonic resonance at $\sim 175 \mathrm{kHz}$. This half resonance is also predicted by the Mathieu equations.

The scaling of the POPS resonance frequency has been measured as a function of the potential well depth and the ion mass. ${ }^{19}$ The POPS frequency scales as the square root of the potential well divided by ion mass, as shown in Eq. (1),
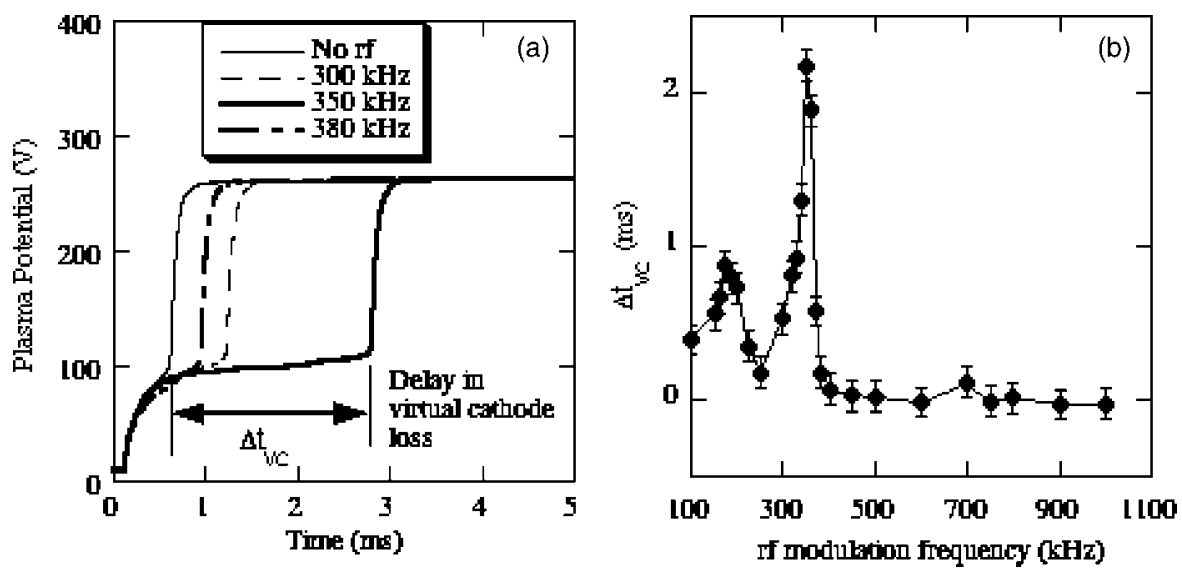

FIG. 3. (a) Temporal evolution of plasma potential at the center of the virtual cathode with and without rf modulation. (Reproduced from Ref. 19.) (b) Delay in the virtual cathode destruction due to rf modulation as a function of modulation frequency. (Reproduced from Ref. 19.) 


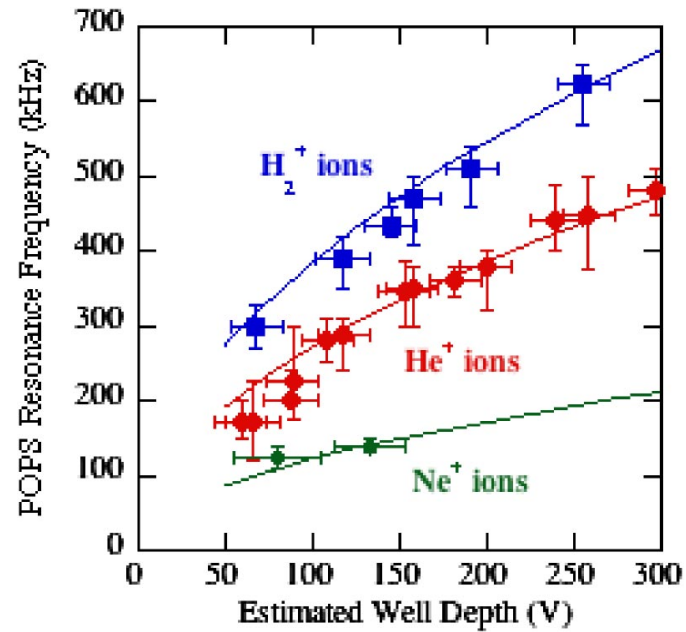

FIG. 4. Comparison between the experimentally measured resonance frequencies due to POPS oscillation (points) and the theoretical calculations (lines) as a function of potential well depth in the virtual cathode and ion mass. (Reproduced from Ref. 19.)

$$
\omega_{\mathrm{POPS}}=\left(\frac{2 e \phi_{00}}{r_{\mathrm{VC}}^{2} m_{i}}\right)^{1 / 2},
$$

where $\omega_{\text {POPS }}$ is the angular frequency of the POPS oscillations, $r_{\mathrm{VC}}$ is the virtual cathode radius, $\phi_{00}$ is the absolute value of the potential well depth in the virtual cathode, $m_{i}$ is the mass of the ion, and $e$ is the electrostatic charge. The well depth of the virtual cathode is controlled by varying the magnitude of the inner grid bias pulse. The fill gas is also varied, generating three different ion species, $\mathrm{H}_{2}^{+}, \mathrm{He}^{+}$, and $\mathrm{Ne}^{+}$. As shown in Fig. 4, excellent agreement between the measured resonance frequencies and theoretical predictions is observed.

The driven POPS oscillation can also be used to control the lifetime of the virtual cathode. As shown in Fig. 5, small

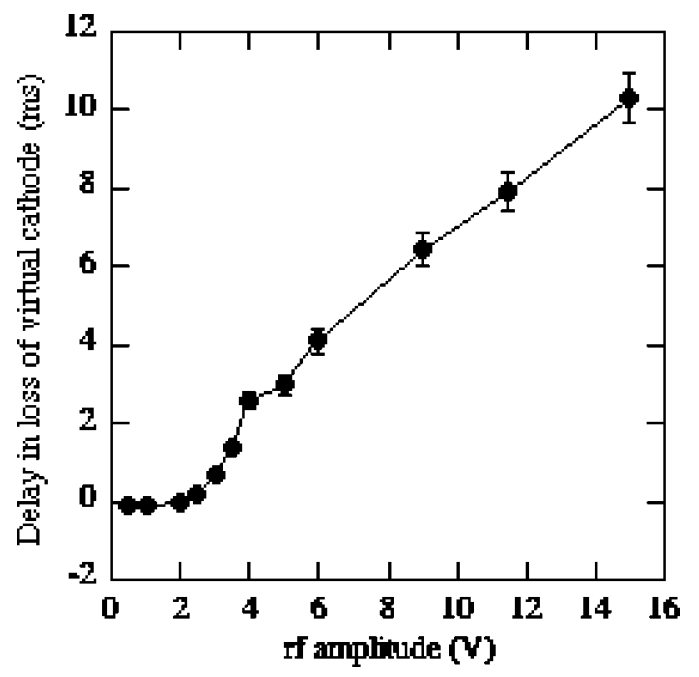

FIG. 5. Delay in the loss of the virtual cathode as a function of rf modulation amplitude at the POPS frequency $\left(280 \mathrm{kHz}\right.$ for $\mathrm{He}^{+}$ions). The inner grid bias of $200 \mathrm{~V}$ (pulse), the outer grid bias of $250 \mathrm{~V}$ (dc), and the extractor grid bias of $150 \mathrm{~V}$ (pulse) are used to create the potential well depth of $\sim 110 \mathrm{~V}$. amplitude perturbations may phase-lock the POPS oscillation but do not enhance the ion ejection enough to balance the ion buildup. Only when the perturbation amplitude exceeds a threshold value can the ion buildup be reduced by the driven POPS oscillations. With increasing rf modulation amplitude, a nearly linear increase in the delay of the virtual cathode decay is observed.

\section{PLASMA COMPRESSION}

One of the most critical issues for the POPS concept is the maximum achievable plasma compression during the POPS collapse phase. The plasma compression is important because it has a large impact on the fusion power. The power density of POPS-based IEC devices can be expressed as ${ }^{15}$

$$
P_{\text {fusion }} \approx \frac{3 f^{2} \varphi_{0}^{2} \theta^{2}}{2 \pi e^{2} r_{\mathrm{vc}}}\langle\sigma v\rangle_{\mathrm{fusion}},
$$

where $f=n_{i} / n_{e}$ is the average ratio between the ion density and electron density in the virtual cathode (typically $\sim 10 \%$ ), $\phi_{0}$ is the potential well depth, $\theta$ is the radial plasma compression ratio $\left(r_{\max } / r_{\min }\right),\langle\sigma v\rangle_{\text {fusion }}$ is the average fusion reaction rate, and $r_{\mathrm{vc}}$ is the radius of the virtual cathode. Consequently, high plasma compression is needed to achieve a high fusion power density and thus make the POPS concept economically attractive. One factor that affects the compression is the extent of space charge neutralization during the ion collapse phase of POPS oscillation. Inadequate space charge neutralization can cause self-repulsion of the ion cloud and limit the plasma compression.

Theoretical studies utilizing a self-consistent particle simulation code that is one dimensional (spherically symmetric) in space and two dimensional in velocity space have been conducted. The code calculates the electric fields using Gauss's law. Particle positions are advanced with a timecentered algorithm. ${ }^{13}$ The edge of the computational domain is the inner grid, where inwardly moving electrons are continually injected and outwardly moving electrons are removed from the simulation. Boundary conditions include the current density, the energy distribution and the angular momentum distribution of the injected electrons.

The code has been used previously to investigate the stability of virtual cathodes formed by electron injection in spherical geometry. ${ }^{13}$ Prior to those studies, work using fluid electrons in a slab geometry ${ }^{21}$ had indicated that the electronelectron two-stream instability would limit the maximum achievable potential well depth to $\sim 14 \%$ of the applied voltage in the case of a constant electron density profile for POPS. However, experimental results indicated that potential wells as deep as $60 \%$ of the applied voltage could be achieved. ${ }^{12,13}$ A detailed study of two-stream stability in a sphere using the one-dimensional (1D) particle code showed that the two-stream stability was much more favorable in spherical geometry than in slab geometry and agreed with the experimental observations. 13

In previous works, ${ }^{14-16}$ it has been assumed that a sufficient number of electrons will follow the ions inward as the ion cloud collapses to provide the desired space charge neutralization. In this paper, the 1D particle code is used to test 

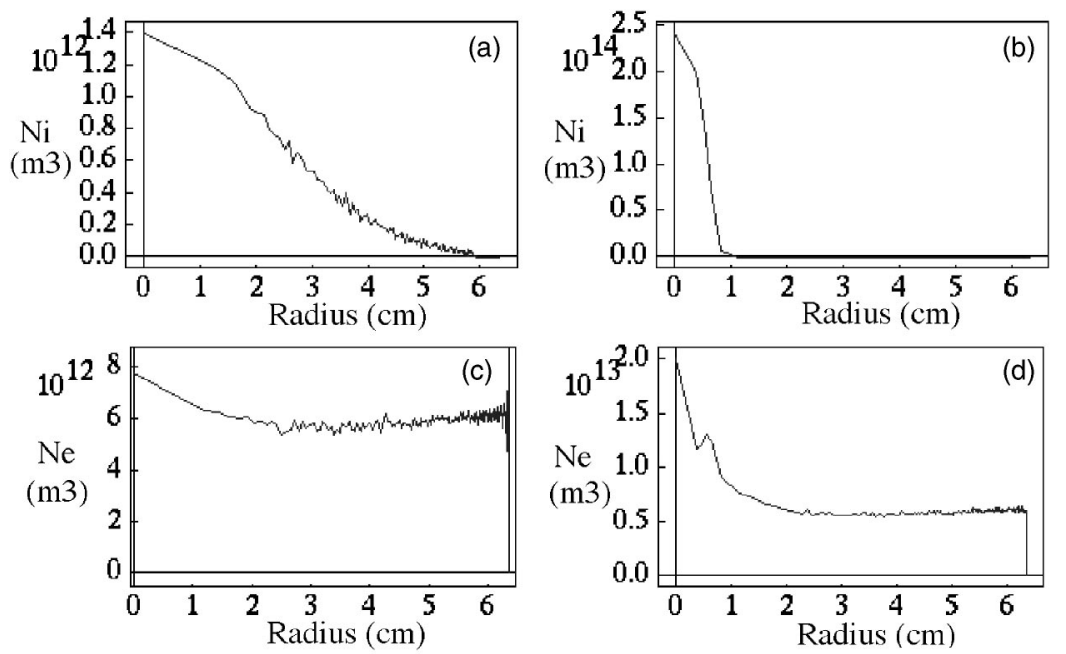

FIG. 6. Ion density profiles: (a) before compression, (b) after compression. Electron density profiles: (c) before compression, (d) after compression. Electrostatic potential profiles: (e) before compression, (f) after compression.
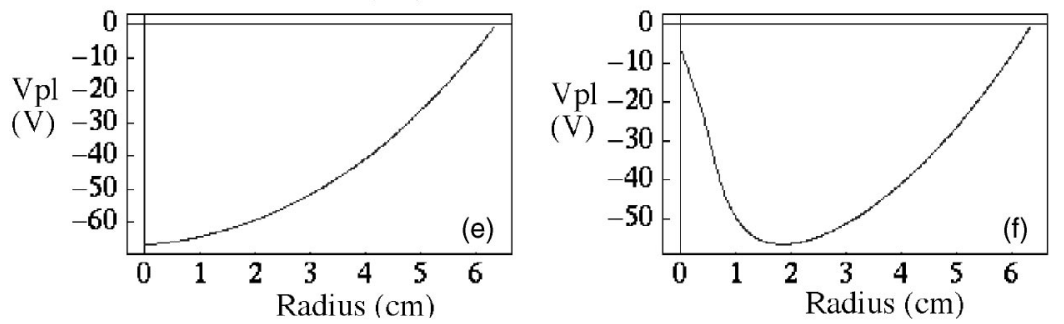

the degree to which this occurs. The ion density profiles before and after compression during a POPS oscillation are shown in Figs. 6(a) and 6(b). For this case, the injected electron distribution function is held constant in time. As expected, the ion density at the center of the sphere increases by more than two orders of magnitude, to almost 2.5 $\times 10^{14} \mathrm{~m}^{-3}$. The radial compression ratio $\left(r_{\text {max }} / r_{\text {min }}\right)$ is about 6.3. However, it is found that most of the electrons cannot follow the ions to the center due to their angular momentum. As shown in Figs. 6(c) and 6(d), the electron density is almost an order of magnitude lower than the ion density at the center, though the electron density has tripled during the POPS collapse. This insufficient space-charge neutralization strongly distorts the plasma potential as shown in Figs. 6(e) and 6(f). The accumulation of ions in the center leads to ion repulsion; hence, the plasma compression is reduced.

A simple remedy has been tested using time-varying boundary conditions. The electron energy, current density, and velocity distribution have been modulated at the POPS frequency in order to focus the electron injection at the center during the POPS collapse. By increasing the electron density at the center during the POPS collapse, as shown in Fig. 7(a), it is possible to improve the space-charge neutralization. As a result, the ion repulsion is mitigated and the plasma compression is greatly enhanced, as shown in Fig. 7(b). The ion density at the center increases by almost two orders of magnitude, compared with the ion density from the fixed boundary conditions, resulting in a radial compression ratio of 23.3 .

\section{DISCUSSION AND CONCLUSIONS}

A critical advantage for a POPS based fusion device is its favorable fusion power density scaling, which increases with the inverse of the virtual cathode radius, as shown in Eq. (2). This attribute provides an economical development path for the POPS concept since each next generation device will be smaller than the previous one. It also leads to a modular, high mass power density device that should be economically competitive. Table I summarizes potential applications of POPS fusion devices depending on the achievable compression, based on the use of $1 \mathrm{~cm}$ radius POPS modules operating with a $100 \mathrm{kV}$ potential well. It is noted that even a fusion power plant or advanced space propulsion can be considered if breakeven is achieved.

The experimental observation of POPS oscillations has provided a critical scientific foundation for further exploration of this promising fusion device concept. In addition,
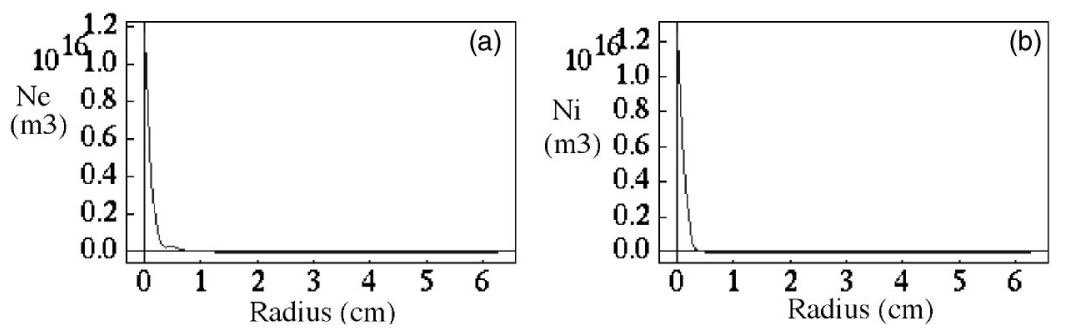

FIG. 7. POPS dynamics for focused electron injection: (a) electron density at maximum compression and (b) ion density at maximum compression. 
TABLE I. Potential applications for POPS fusion devices and required radial compression based on modular design.

\begin{tabular}{ccccc}
\hline \hline Fuel & Applications & Compression ratio $\left(r_{\max } / r_{\min }\right)$ & Neutron rate & Modules \\
\hline D-D & Active nuclear assay & 26 or 10 for seven modules & $\sim 1.0 \times 10^{11} \mathrm{n} / \mathrm{s}$ & 1 \\
D-D & Neutron tomography & 17 & $\sim 1.0 \times 10^{12} \mathrm{n} / \mathrm{s}$ & 25 \\
D-D & PET isotope production & 70 & $\sim 1.0 \times 10^{15} \mathrm{n} / \mathrm{s}$ & $\sim 1400$ \\
D-D & Fusion power (100 MW) & 1000 & $\sim 1.0 \times 10^{20} \mathrm{n} / \mathrm{s}$ & $\sim 1.1 \times 10^{7}$ \\
D-T & Fusion power (100 MW) & 86 & $\sim 1.6 \times 10^{20} \mathrm{n} / \mathrm{s}$ & $\sim 2.8 \times 10^{6}$ \\
\hline \hline
\end{tabular}

pulsed power operation and the control of the virtual cathode lifetime by driven POPS oscillations represent important steps toward expanding the POPS operating regime. Still, significant issues remain regarding the high voltage operation of POPS systems. A number of experimental upgrades are being designed to increase the potential well and to overcome the decay of the virtual cathode.

Although space-charge neutralization remains a major issue, a substantial plasma compression has been observed in 1D particle simulations, as shown in Fig. 7. However, a number of factors can further influence the extent of ion cloud compression during POPS. Finite grids and multidimensional perturbations of the background electrons can lead to an asymmetric potential structure. Such irregularities in the potential well will reduce the ion cloud compression. Electronion energy transfer, by collisions or instability, can also increase the ion adiabat and may inhibit high compression ratios. Currently, direct experimental measurement of the plasma compression is being pursued using electron beam diagnostics. In addition a two dimensional particle-in-cell code is being implemented to address multidimensional effects on POPS compression. ${ }^{22,23}$

The required compression ratio would be much smaller if deuterium and tritium, rather than deuterium alone, could be used to produce fusion reactions. This will require the two different ions species to collapse simultaneously. Due to their mass ratio, the phases of deuterium and tritium will closely overlap every six deuterium periods. Thus, one can use the fractional harmonics in external modulation to excite the POPS oscillation, as shown in Fig. 5. Conversely, one can also drive the POPS oscillation at the fundamental frequency of deuterium, which will create a near simultaneous collapse every six deuterium periods. Since the increased cross section of the D-T fusion yield is about 200 over the D-D yield, a net gain of 20-30 may be achievable by dynamically controlling the amplitude and frequencies of the external modulation.

In summary, POPS is a promising novel fusion device concept that can greatly increase the fusion yield over existing IEC schemes. It has an attractive development path and its implementation is compatible to a modular, high mass power density device that should be economically competitive. The experimental confirmation of the POPS demonstrates the validity of the POPS concept. The 1D particle code shows that significant plasma compression can be achieved by using the dynamic control of electron injection to improve the space-charge neutralization. Depending on the achievable plasma compression, a number of practical applications appear to be promising. Finally, a number of important issues facing the further exploration of POPS concept have been presented.

\section{ACKNOWLEDGMENTS}

The authors gratefully thank Dr. Martin Taccetti, Dr. Martin Schauer, and Dr. Carter Munson of Los Alamos National Laboratory for their technical advice on the POPS experiment.

This work was supported by U.S. Department of Energy Contract No. W-7405-ENG-36.

${ }^{1}$ W. C. Elmore, J. L. Tuck, and K. M. Watson, Phys. Fluids 2, 239 (1959).

${ }^{2} \mathrm{P}$. T. Farnsworth, "Electric discharge device for producing interactions between nucleii," U.S. Patent No. 3,358,402 (28 June 1966).

${ }^{3}$ R. L. Hirsch and G. A. Meeks, "Apparatus for generating fusion reactions," U.S. Patent No. 3,530,497 (22 September 1970).

${ }^{4}$ R. L. Hirsch, J. Appl. Phys. 38, 4522 (1967).

${ }^{5}$ R. W. Bussard, Fusion Technol. 19, 273 (1991).

${ }^{6}$ T. B. Mitchell, M. M. Schauer, and D. C. Barnes, Phys. Rev. Lett. 78, 58 (1997).

${ }^{7}$ D. C. Barnes, T. B. Mitchell, and M. M. Schauer, Phys. Plasmas 4, 1745 (1997).

${ }^{8}$ M. M. Schauer, T. B. Mitchell, M. H. Holzscheiter, and D. C. Barnes, Rev. Sci. Instrum. 68, 3340 (1997).

${ }^{9}$ W. M. Nevins, Phys. Plasmas 2, 3804 (1995).

${ }^{10}$ T. H. Rider, Phys. Plasmas 2, 1853 (1995).

${ }^{11}$ L. Chacon, G. H. Miley, D. C. Barnes, and D. A. Knoll, Phys. Plasmas 7, 4547 (2000).

${ }^{12}$ J. Park, R. A. Nebel, W. G. Rellergert, and M. D. Sekora, Phys. Plasmas 10, 3841 (2003).

${ }^{13}$ R. A. Nebel, S. Stange, J. Park, J. M. Taccetti, S. K. Murali, and C. E. Garcia, Phys. Plasmas 12, 012701 (2005).

${ }^{14}$ D. C. Barnes and R. A. Nebel, Phys. Plasmas 5, 2498 (1998).

${ }^{15}$ R. A. Nebel and D. C. Barnes, Fusion Technol. 38, 28 (1998).

${ }^{16}$ R. A. Nebel and J. M. Finn, Phys. Plasmas 7, 839 (2000).

${ }^{17}$ A. Siebenförcher and R. Schrittwieser, Rev. Sci. Instrum. 67, 849 (1996).

${ }^{18}$ J. R. Smith, N. Hershkowitz, and P. Coakley, Rev. Sci. Instrum. 50, 210 (1979).

${ }^{19}$ J. Park, R. A. Nebel, S. Stange, and S. Krupakar Murali, "Experimental observation of the Periodically Oscillating Plasma Sphere (POPS) oscillation in a gridded inertial electrostatic confinement device," Phys. Rev. Lett. (submitted)

${ }^{20}$ See, for instance, M. Abramowitz and I. A. Stegun, Handbook of Mathematical Functions (Dover, New York, 1965), Chap. 20, p. 721.

${ }^{21}$ R. A. Nebel and J. M. Finn, Phys. Plasmas 8, 1505 (2001).

${ }^{22}$ G. Lapenta, J. Comput. Phys. 193, 159 (2003).

${ }^{23}$ J. U. Brackbill and D. W. Forslund, in Multiple Time Scales, Simulation of Low-Frequency Electromagnetic Phenomena in Plasmas, edited by J. U. Brackbill and B. I. Cohen (Academic, San Diego, 1985), p. 271. 